\title{
Effects of the Switching From High-Purity Eicosapentaenoic Acid to Combination of Eicosapentaenoic Acid and Docosahexaenoic Acid on Metabolic Parameters: A Retrospective Longitudinal Study
}

\author{
Hisayuki Katsuyama $^{\text {a }}$, Shohei Matsuura ${ }^{\text {a }}$, Hidekatsu Yanaia, b
}

\begin{abstract}
Background: Effects of the switching from high-purity eicosapentaenoic acid ester (hp-EPA) to n-3 fatty acid formulation containing EPA and docosahexaenoic acid (DHA) on metabolic parameters remain largely unknown.
\end{abstract}

Methods: We retrospectively picked up patients who had been prescribed EPA + DHA for 2 months or longer after the prescription of hp-EPA for 2 months or longer between January 2010 and December 2015. We compared the data at baseline and at 2 months after the switching from hp-EPA to EPA + DHA.

Results: Thirty-six patients were eligible for the analyses in our study. Serum triglyceride (TG) showed a non-significant decrease by approximately $9 \%$ after the switching. Serum TG showed a nonsignificant decrease by $24 \%$ and $10 \%$, in the switching to the formulation including half daily dose of EPA and DHA and the formulation including the same daily dose of EPA and DHA, respectively. In patients who had shown a decrease in TG after the switching, serum TG at baseline was higher than that in patients who had shown an increase and non-change in TG after the switching. Further, in patients who had shown a decrease in TG after the switching to the formulation including the same daily dose of EPA and DHA, serum TG was significantly higher than that in patients who had shown an increase and non-change in TG. In the analysis of all patients, in patients with baseline $\mathrm{TG} \geq 150 \mathrm{mg} / \mathrm{dL}$, TG tended to decrease. In the analysis of patients who underwent the switching to the formulation including the same daily dose of EPA and DHA, in patients with baseline TG $\geq$ $150 \mathrm{mg} / \mathrm{dL}$, TG significantly decreased.

Conclusion: We studied effects of the switching from hp-EPA to EPA + DHA on metabolic parameters, and found that the switching is more

Manuscript accepted for publication May 03, 2016

${ }^{a}$ Department of Internal Medicine, National Center for Global Health and Medicine Kohnodai Hospital, Chiba, Japan

${ }^{\mathrm{b}}$ Corresponding Author: Hidekatsu Yanai, Department of Internal Medicine, National Center for Global Health and Medicine Kohnodai Hospital, 1-7-1 Kohnodai, Ichikawa, Chiba 272-0034, Japan.

Email: dyanai@hospk.ncgm.go.jp

doi: http://dx.doi.org/10.14740/jem348w effective to reduce TG in patients with higher TG levels at baseline.

Keywords: Coronary risk factors; Docosahexaenoic acid; Eicosapentaenoic acid; Triglyceride

\section{Introduction}

n-3 polyunsaturated fatty acids (FAs) have been reported to be effective in reducing the risk of cardiovascular diseases [1-3]. In Japan, compared with a modest fish intake of once a week and/or $20 \mathrm{~g} /$ day, a higher intake was associated with reduced risk of coronary heart disease among middle-aged persons, indicating an effectiveness of fish oil including eicosapentaenoic acid (EPA) and docosahexaenoic acid (DHA) for prevention of coronary heart disease [4]. We previously reviewed the articles about effects of fish oils intake on high-density lipoprotein (HDL) metabolism, and concluded that fish oils consumption, especially DHA consumption, may be favorably associated with HDL metabolism [5]. Further, we reviewed the articles about effects of intake of fish or fish oils on the development of diabetes, and concluded marine $\mathrm{n}-3$ polyunsaturated FAs have beneficial effects on the prevention of type 2 diabetes in Asian populations [6]. Accumulated data have suggested that n-3 FA prevents cardiovascular diseases and improves coronary risk factors. However, it remains unknown which FA improves coronary risk factors more, EPA or DHA. High-purity EPA ester (hp-EPA) and n-3 FA formulation containing $465 \mathrm{mg}$ of EPA and $375 \mathrm{mg}$ of DHA per gram (EPA + DHA) have been approved and widely used to date in Japan. Here, we studied effects of the switching from hp-EPA to EPA + DHA on metabolic parameters.

\section{Materials and Methods}

\section{Subjects}

This study was approval by the Institutional Ethics Committee in National Center for Global Health and Medicine (NCGMG-001909-00), and was also performed in accordance with the Declaration of Helsinki. 
Table 1. Baseline Characteristics of the Study Subjects $(n=$ 36)

\begin{tabular}{ll}
\hline Age (years) & $63.8 \pm 16.4$ \\
Sex (male/female) & $21 / 15$ \\
Body height $(\mathrm{cm})$ & $160.4 \pm 9.8$ \\
Body weight $(\mathrm{kg})$ & $66.2 \pm 14.9$ \\
Body mass index (BMI) $\left(\mathrm{kg} / \mathrm{m}^{2}\right)$ & $26.3 \pm 5.4$ \\
Patients taking anti-diabetic drugs (n) & 14 \\
Patients taking anti-hypertensive drugs $(\mathrm{n})$ & 19 \\
Patients taking anti-platelet agents $(\mathrm{n})$ & 9 \\
Patients taking lipid modifying agents $(\mathrm{n})$ & 35 \\
Statins & 17 \\
Ion-exchange resin & 2 \\
Fibrates & 4 \\
Ezetimibe & 12 \\
\hline
\end{tabular}

We selected patients who had been prescribed EPA + DHA for 2 months or longer after prescription of hp-EPA for 2 months or longer between January 2010 and December 2015, based on medical charts. We compared the data at baseline and at 2 months after the switching from hp-EPA to EPA + DHA. Body weight, HbA1c, serum total cholesterol (TC), low-density lipoprotein-cholesterol (LDL-C), triglyceride (TG), highdensity lipoprotein-cholesterol (HDL-C), non-HDL-C, aspartate aminotransferase (AST) and alanine aminotransferase (ALT) were measured almost at the same time point at the baseline and at 2 months after the switching.

Comparison of the variables determined before and after was analyzed by a paired Student's $t$-test. All data were expressed as mean $\pm \mathrm{SD} . \mathrm{P}<0.05$ and $\mathrm{P}<0.01$ were considered to be statistically significant and have a tendency, respectively.

\section{Results}

We found 526 and 130 patients who had taken hp-EPA and EPA + DHA at least once between January 2010 and December
Table 2. Changes in Daily EPA Dose by the Switching From High-Purity EPA to EPA + DHA

\begin{tabular}{|lll}
\hline $\begin{array}{l}\text { Daily EPA dose before } \\
\text { switching (mg/day) }\end{array}$ & $\begin{array}{l}\text { Daily EPA dose after } \\
\text { switching (mg/day) }\end{array}$ & $\mathbf{n}$ \\
\hline 600 & 930 & 1 \\
600 & 1,860 & 1 \\
900 & 930 & 4 \\
\hline 1,200 & 930 & 1 \\
1,800 & 930 & 8 \\
1,800 & 1,860 & 17 \\
2,700 & 930 & 2 \\
\hline 2,700 & 1,860 & 1 \\
\hline
\end{tabular}

2015, respectively. Fifty-eight patients had taken both hp-EPA and EPA + DHA, and 36 patients had undergone the switching from hp-EPA to EPA + DHA.

Table 1 shows the baseline characteristics of the studied subjects. Table 2 shows the changes in daily EPA dose by the switching from hp-EPA to EPA + DHA. Table 3 shows effects of the switching from hp-EPA to EPA + DHA on metabolic parameters. Body weight, TC, HDL-C, LDL-C, non-HDL-C, AST, ALT and HbAlc almost did not change. TG showed a nonsignificant decrease by approximately $9 \%$ after the switching.

We divided patients into two subgroups: patients underwent the switching from hp-EPA (1,800 mg/day) to EPA $(930 \mathrm{mg} /$ day) + DHA (750 mg/day) which is the switching to the formulation including half dose of EPA and DHA (half EPA + DHA) $(\mathrm{n}=8)$ and patients underwent the switching from EPA (900 and $1,800 \mathrm{mg}$ /day) to EPA (930 and 1,860 mg/day) + DHA (750 and $1,500 \mathrm{mg} /$ day) which is the switching to the formulation including the same daily dose of EPA and DHA (same EPA + DHA) $(n=21)$. In both groups, metabolic parameters except for TG almost did not change (Tables 4 and 5). Serum TG showed a non-significant decrease by $24 \%$ and $10 \%$ in the switching to the half EPA + DHA and the same EPA + DHA, respectively.

Comparison of serum TG at baseline in subgroups which were divided by effect of the switching from hp-EPA to EPA + DHA on serum TG was shown in Table 6. In patients who

Table 3. Effect of the Switching From High-Purity EPA to EPA + DHA on Metabolic Parameters in All Subjects $(n=36)$

\begin{tabular}{lllll}
\hline & n & Before & 2 months after & P value \\
\hline Body weight (kg) & 17 & $66.2 \pm 16.7$ & $66.6 \pm 17.1$ & 0.73 \\
Total cholesterol (mg/dL) & 26 & $192 \pm 36$ & $192 \pm 38$ & 0.77 \\
HDL-cholesterol (mg/dL) & 32 & $48 \pm 14$ & $49 \pm 15$ & 0.78 \\
LDL-cholesterol (mg/dL) & 21 & $108 \pm 36$ & $109 \pm 33$ & 0.68 \\
Triglyceride (mg/dL) & 34 & $180 \pm 136$ & $163 \pm 102$ & 0.23 \\
Non-HDL-cholesterol (mg/dL) & 24 & $138 \pm 29$ & $141 \pm 36$ & 0.87 \\
AST (IU/L) & 34 & $27 \pm 17$ & $28 \pm 14$ & 0.34 \\
ALT (IU/L) & 32 & $31 \pm 21$ & $32 \pm 22$ & 0.35 \\
HbA1c (\%) & 29 & $6.4 \pm 0.8$ & $6.4 \pm 0.7$ & 0.22 \\
\hline
\end{tabular}


Table 4. Effect of the Switching From High-Purity EPA $(1,800 \mathrm{mg} /$ day $)$ to EPA ( $930 \mathrm{mg} /$ day $)$ + DHA (750 mg/day), the Switching to the Formulation Including Half Daily Dose of EPA and DHA, on Metabolic Parameters $(n=8)$

\begin{tabular}{lllll}
\hline & n & Before & 2 months after & P value \\
\hline Body weight (kg) & 2 & $57.5 \pm 6.5$ & $57.9 \pm 7.2$ & 0.69 \\
Total cholesterol (mg/dL) & 6 & $199 \pm 23$ & $198 \pm 31$ & 0.87 \\
HDL-cholesterol (mg/dL) & 6 & $52 \pm 10$ & $53 \pm 10$ & 0.80 \\
LDL-cholesterol (mg/dL) & 6 & $118 \pm 43$ & $120 \pm 45$ & 0.85 \\
Triglyceride (mg/dL) & 7 & $191 \pm 196$ & $146 \pm 101$ & 0.41 \\
Non-HDL-cholesterol (mg/dL) & 5 & $142 \pm 29$ & $148 \pm 41$ & 0.73 \\
AST (IU/L) & 7 & $30 \pm 27$ & $34 \pm 30$ & 0.53 \\
ALT (IU/L) & 7 & $36 \pm 30$ & $34 \pm 30$ & 0.47 \\
HbA1c (\%) & 4 & $6.2 \pm 0.5$ & $6.2 \pm 0.5$ & 0.49 \\
\hline
\end{tabular}

Table 5. Effect of the Switching From High-Purity EPA (900 and 1,800 mg/day) to EPA (930 and $1,860 \mathrm{mg} /$ day $)+$ DHA (750 and 1,500 mg/day), the Switching to the Formulation Including the Same Daily Dose of EPA and DHA, on Metabolic Parameters $(n=21)$

\begin{tabular}{lllll} 
& n & Before & 2 months after & P value \\
\hline Body weight $(\mathrm{kg})$ & 12 & $57.5 \pm 6.5$ & $57.9 \pm 7.2$ & 0.69 \\
Total cholesterol (mg/dL) & 15 & $199 \pm 23$ & $198 \pm 31$ & 0.87 \\
HDL-cholesterol (mg/dL) & 15 & $52 \pm 10$ & $53 \pm 10$ & 0.80 \\
LDL-cholesterol (mg/dL) & 11 & $118 \pm 43$ & $120 \pm 45$ & 0.85 \\
Triglyceride (mg/dL) & 22 & $176 \pm 119$ & $159 \pm 101$ & 0.22 \\
Non-HDL-cholesterol (mg/dL) & 15 & $142 \pm 29$ & $148 \pm 41$ & 0.73 \\
AST (IU/L) & 21 & $30 \pm 27$ & $34 \pm 30$ & 0.53 \\
ALT (IU/L) & 21 & $36 \pm 30$ & $34 \pm 30$ & 0.47 \\
HbA1c (\%) & 21 & $6.2 \pm 0.5$ & $6.2 \pm 0.5$ & 0.49 \\
\hline
\end{tabular}

had shown a decrease in TG after the switching, serum TG at baseline was higher than that in patients who had shown an increase and non-change in TG after the switching. Further, in patients who had shown a decrease in TG after the switching to the same EPA + DHA, serum TG was significantly higher than that in patients who had shown an increase and non-change in TG after the switching.

Effects of the switching from hp-EPA to EPA + DHA on serum TG in subgroups which were divided by baseline TG ( $\mathrm{TG} \geq 150 \mathrm{mg} / \mathrm{dL}$ or $\mathrm{TG}<150 \mathrm{mg} / \mathrm{dL}$ ) were shown in Table 7 .
In the analysis of all patients, in patients with baseline $\mathrm{TG} \geq 150$ $\mathrm{mg} / \mathrm{dL}, \mathrm{TG}$ tended to decrease. In the analysis of patients who underwent the switching to the same EPA + DHA, in patients with baseline $\mathrm{TG} \geq 150 \mathrm{mg} / \mathrm{dL}$, TG significantly decreased.

\section{Discussion}

n-3 FAs have several beneficial cardiovascular protective properties including TG-reducing ability. In the type IIb hy-

Table 6. Comparison of Serum Triglyceride (TG) at Baseline in Subgroups Which Were Divided by Effect of the Switching From High-Purity EPA to EPA + DHA on Serum TG

\begin{tabular}{|c|c|c|c|c|}
\hline & $\mathbf{n}$ & TG decreased & $\begin{array}{l}\text { TG increased and } \\
\text { TG did not change }\end{array}$ & P value \\
\hline All patients & 34 & $214 \pm 152(\mathrm{n}=22)$ & $119 \pm 51(\mathrm{n}=12)$ & 0.051 \\
\hline $\begin{array}{l}\text { Patients underwent the switching to the formation including the same daily dose } \\
\text { of EPA and DHA (EPA } \rightarrow \text { same EPA }+ \text { DHA) }\end{array}$ & 21 & $212 \pm 129(n=14)$ & $103 \pm 25(\mathrm{n}=7)$ & 0.049 \\
\hline
\end{tabular}


Table 7. Effect of the Switching From High-Purity EPA to EPA + DHA on Serum TG in Subgroups Which Were Divided by Baseline TG

\begin{tabular}{|cllll|}
\hline & n & Before & 2 months after & P value \\
\hline All patients & 34 & & & \\
TG $\geq 150 \mathrm{mg} / \mathrm{dL}$ & 14 & $298 \pm 140$ & $249 \pm 91$ & 0.096 \\
$\mathrm{TG}<150 \mathrm{mg} / \mathrm{dL}$ & 20 & $99 \pm 24$ & $104 \pm 54$ & 0.722 \\
$\mathrm{EPA} \rightarrow$ half EPA + DHA & 8 & & & 0.549 \\
$\mathrm{TG} \geq 150 \mathrm{mg} / \mathrm{dL}$ & 2 & $417 \pm 248$ & $273 \pm 80$ & 0.907 \\
$\mathrm{TG}<150 \mathrm{mg} / \mathrm{dL}$ & 5 & $101 \pm 23$ & $97 \pm 54$ & \\
$\mathrm{EPA} \rightarrow \mathrm{same} \mathrm{EPA}+\mathrm{DHA}$ & 21 & & & 0.009 \\
$\mathrm{TG} \geq 150 \mathrm{mg} / \mathrm{dL}$ & 8 & $297 \pm 114$ & $239 \pm 107$ & 0.673 \\
\hline $\mathrm{TG}<150 \mathrm{mg} / \mathrm{dL}$ & 13 & $102 \pm 20$ & $110 \pm 56$ & \\
\hline
\end{tabular}

perlipidemic patients, the fish-oil diet led to decreases in both plasma TC (-27\%) and TG (-64\%), as compared with the control diet. Very-low-density lipoprotein (VLDL) was remarkably reduced [7]. With fish oil, the type V hyperlipidemic patients had marked decreases in TC and TG levels $(-45 \%$ and $-79 \%$, respectively), and VLDL levels were also lowered [7]. Metabolically controlled trials in which large amounts of fish oil were fed to normal volunteers and hyperlipidemic patients showed that n-3 FAs are effective at lowering plasma TC and TG levels [8]. Although more recent trials using smaller, more practical doses of fish oil supplements have confirmed the TGreducing effect, however, they have shown little effect on TC levels; hypertriglyceridemic patients have shown increases in LDL-C levels while taking n-3 FA supplements [8]. Discrepancies among fish oil studies regarding the effects of n-3 FA on LDL-C levels exist [8].

In the double-blind, randomized crossover study which investigated the effects of 6-week treatment with n-3 FA esters (4 g/day) on serum lipids [9], n-3 FA induced a modest increase from baseline in LDL-C (3.4\%), a significant decrease in VLDL (-18.8\%), TG (-18.7\%), and a significant increase in HDL-C (3.3\%). However, in Japan EPA lipid intervention study (JELIS) which used hp-EPA [10], TC, LDL-C and TG significantly decreased by $19 \%, 25 \%$ and $9 \%$, respectively, and only small increase in HDL-C was observed.

Since hp-EPA and EPA + DHA have been approved and widely used to date in Japan, we studied effects of the switching from hp-EPA to EPA + DHA. TG showed a non-significant decrease by approximately $9 \%$ after the switching. Serum TG showed a non-significant decrease by $24 \%$ and $10 \%$ in the switching to the half EPA + DHA and the same EPA + DHA, respectively. Further, our study demonstrated that the switching from hp-EPA to EPA + DHA is more effective to reduce TG in patients with higher TG levels at baseline.

To our knowledge, the present study is the first to show effects of the switching from hp-EPA to EPA + DHA because countries except for Japan where both hp-EPA and EPA + DHA are available, are very limited. We found a case report showing the switching from EPA + DHA to hp-EPA [11]. In the case, the switching produced a decrease in TC, LDL-C, TG and nonHDL-C, by $34 \%, 28 \%, 41 \%$ and $44 \%$, respectively, challeng- ing our result. However, in our study, 12 of 34 (35\%) patients showed an increase and non-change of TG by the switching from hp-EPA to EPA + DHA, suggesting an importance of accumulation of studied subjects.

Present study has several limitations. First, other hypoglycemic, anti-hypertensive, or lipid lowering agents, food intakes and/or exercise levels may have an influence on the study results. Second, the number of studied subjects was small because of the limited availability. Third, since the study was retrospective and based on medical charts, lack of data might influence the results. A more detailed prospective study is recommended to evaluate the effects of switching from hp-EPA to $\mathrm{EPA}+\mathrm{DHA}$ on metabolic parameters more validly.

\section{Conclusion}

We studied effects of the switching from hp-EPA to EPA + DHA on metabolic parameters, and found that the switching is more effective to reduce TG in patients with higher TG levels at baseline.

\section{Author Contributions}

HK, SM and HY designed the research. HK and SM collected data. HK, SM and HY analyzed data, and wrote the paper. All authors read and approved the final paper.

\section{Conflicts of Interest}

The authors declare that they have no conflicts of interest concerning this article.

\section{References}

1. Kromhout D, Bosschieter EB, de Lezenne Coulander C. The inverse relation between fish consumption and 20year mortality from coronary heart disease. $\mathrm{N}$ Engl $\mathrm{J}$ 
Med. 1985;312(19):1205-1209.

2. Burr ML, Fehily AM, Gilbert JF, Rogers S, Holliday RM, Sweetnam PM, Elwood PC, et al. Effects of changes in fat, fish, and fibre intakes on death and myocardial reinfarction: diet and reinfarction trial (DART). Lancet. 1989;2(8666):757-761.

3. Daviglus ML, Stamler J, Orencia AJ, Dyer AR, Liu K, Greenland P, Walsh MK, et al. Fish consumption and the 30 -year risk of fatal myocardial infarction. N Engl J Med. 1997;336(15):1046-1053.

4. Iso H, Kobayashi M, Ishihara J, Sasaki S, Okada K, Kita Y, Kokubo Y, et al. Intake of fish and n3 fatty acids and risk of coronary heart disease among Japanese: the Japan Public Health Center-Based (JPHC) Study Cohort I. Circulation. 2006;113(2):195-202.

5. Yanai H, Katsuyama H, Hamasaki $\mathrm{H}$, Abe S, Tada N, Sako A. Effects of Dietary Fat Intake on HDL Metabolism. J Clin Med Res. 2015;7(3):145-149.

6. Yanai H, Hamasaki H, Katsuyama H, Adachi H, Moriyama S, Sako A. Effects of intake of fish or fish oils on the development of diabetes. J Clin Med Res. 2015;7(1):812.
7. Phillipson BE, Rothrock DW, Connor WE, Harris WS, Illingworth DR. Reduction of plasma lipids, lipoproteins, and apoproteins by dietary fish oils in patients with hypertriglyceridemia. N Engl J Med. 1985;312(19):1210-1216.

8. Harris WS. Fish oils and plasma lipid and lipoprotein metabolism in humans: a critical review. J Lipid Res. 1989;30(6):785-807.

9. Maki KC, Lawless AL, Kelley KM, Dicklin MR, Kaden VN, Schild AL, Rains TM, et al. Effects of prescription omega-3-acid ethyl esters on fasting lipid profile in subjects with primary hypercholesterolemia. J Cardiovasc Pharmacol. 2011;57(4):489-494.

10. Yokoyama M, Origasa H, Matsuzaki M, Matsuzawa $Y$, Saito Y, Ishikawa Y, Oikawa S, et al. Effects of eicosapentaenoic acid on major coronary events in hypercholesterolaemic patients (JELIS): a randomised open-label, blinded endpoint analysis. Lancet. 2007;369(9567):10901098.

11. Kedia AW, Lynch E. Effects of switching from omega3 -acid ethyl esters to icosapent ethyl in a statin-treated patient with elevated triglycerides. Postgrad Med. 2015;127(8):869-873. 